\title{
A Novel Approach for Brain Tumor Detection Using MRI Images
}

\author{
Abd El Kader Isselmou, Shuai Zhang, Guizhi Xu \\ Province-Ministry Joint Key Laboratory of Electromagnetic Field and Electrical Apparatus Reliability, Department of Biomedical \\ Engineering, Hebei University of Technology, Tianjin, China \\ Email: isselmoukader@outlook.com, zs@hebut.edu.cn, gzxu@hebut.edu.cn
}

How to cite this paper: Isselmou, A. El K., Zhang, S. and Xu, G.Z. (2016) A Novel Approach for Brain Tumor Detection Using MRI Images. J. Biomedical Science and Engineering, 9, 44-52.

http://dx.doi.org/10.4236/jbise.2016.910B006

Received: July 28, 2016

Accepted: September 20, 2016

Published: September 23, 2016

\begin{abstract}
Processing magnetic resonance images are very complex and constantly studied by the researchers to give doctors better ability to diagnose the patients. In order to detect automatically suspicious regions or tumors, we present a new approach inspired by threshold segmentation and based on morphological operations in this paper. The advantages of our approach come from the complementarities between these two approaches. The morphological operations extract roughly the tumor region and eventually can affect healthy while the threshold segmentation method gives a clear picture of the structure of the different brain and therefore these two approaches improve significantly the threshold segmentation and detection and extraction of the tumor zone based on morphological operations.
\end{abstract}

\section{Keywords}

MRI, Threshold Segmentation, Morphological Operations, Tumor Identification, Filters

\section{Introduction}

Nowadays, brain tumor is one the main reason for increasing mortality among adults and kids. It's been concluded in the research of the majority of the western world that number of individuals who suffering and dying from brain tumors continues to be increased to 300 a year during past few decades [1]. More than 612,000 Americans will likely be identified as having a brain tumor and also over millions of people in the united states are living with brain tumors that have yet to be detected based on Nation's Brain Tumor Foundation (NBTF) and American Brain Tumor Association (ABTA) [2] where approximately 4300 children younger than age 20 will be informed they have 
primary brain tumors, which 3050 will likely be under age 15 .

As this number is candidate to increase, tools and methods to detect, extract the tumors and also to analyze their behavior are increasingly widespread and must consider the type of tumor, the kind of images to be utilized and depending there from the several approaches to use or develop.

\subsection{Brain Tumor}

The word tumor, also referred to as neoplasm, means the abnormal expansion of the tissues that results when cells divide more than they need to or do not die after they should [3]. Brain tumor can be an abnormal mass of tissue through which cells grow and multiply uncontrollably, seemingly unchecked by the mechanisms that control normal cells. Two large individuals are recognized and which rely on the origin with the tumors (primary, metastatic) in addition to their styles of growth of malignity (benign, malignant).

\subsection{MRI Brain Tumors' Images}

Magnetic Resonance Imaging (MRI) is the most sophisticated and waves used magnetic resonance imaging to obtain high-quality images from all over the body and tissues and to ability of MRI to detect the smallest details within the body. MRI is usually used when treating brain tumors or any other cancers. Through high-resolution imaging magnetic resonance images we can infer anatomical information and find out where abnormalities. This technique has a great ability to detect differences in tissue and structures and is better than computed tomography for the detection of the size of the tumor in the brain [4].

A more classy and versatile approach will depend on thresholding [5] which divides the image into two regions this forming a binarized image a depending on a typical threshold method [6] for segmentation. Its gives the better segmented results over other traditional algorithms because threshold value is dependent on the inner cluster variance.

A morphological filtering [7] [8] based approach of brain tumor segmentation on MRI images provides significant outputs through operations like erosion and dilation. It work over the binarized image for automatic region splitting dependant on region growing approach like automated seeded selection [9] also for detection and extraction of tumor. Before processing the image must be pre-processed by removing noise using fourth order derivative. Also a mixture of flood-fill algorithm with morphological operations also detects and extracts tumor brain MRI image. Rest of the paper is arranged in four parts. Part 2 explains proposed mechanism for brain tumor segmentation and detection in MRI image. Experimental results of proposed framework and calculated overall error and accuracy of the approach are shown in part 3 followed by the conclusion in part 4.

\section{Proposed Approach}

The proposed framework is shown in Figure 1 with detailed internal architecture for 


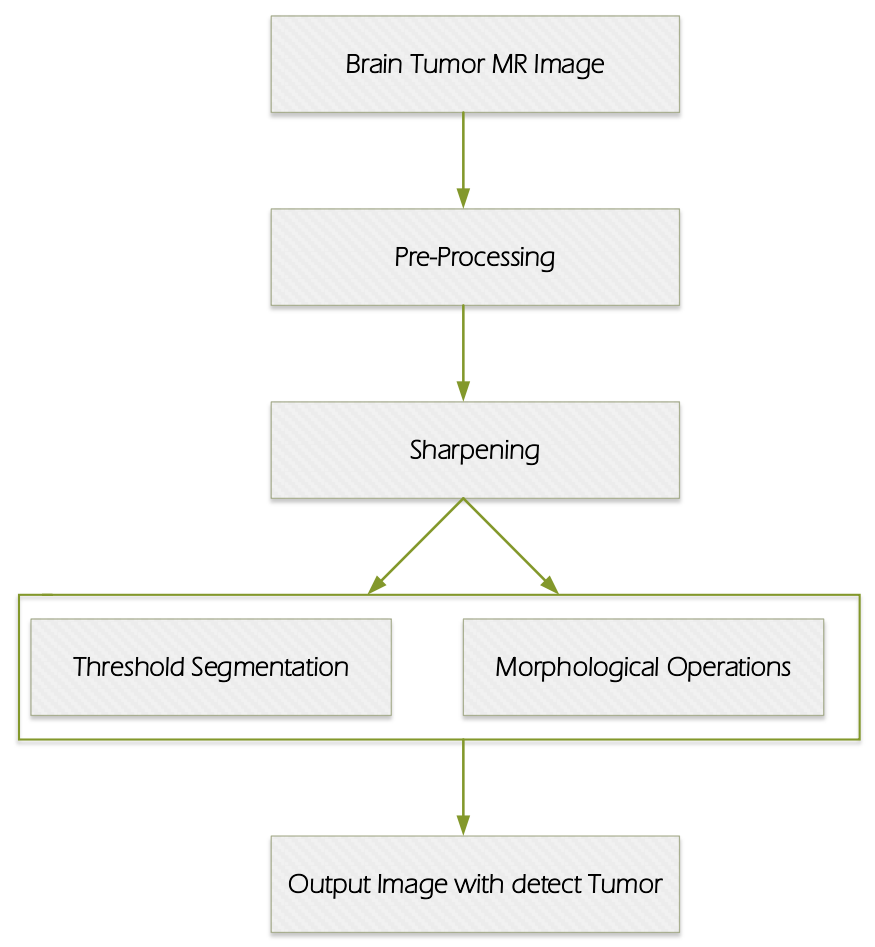

Figure 1. Proposed architecture of the framework.

the detection and extraction of the brain tumor from MR images. The approach consists of three phase such that during first phase input image is being pre-processing followed by second phase threshold segmentation with further application of morphological operations, finally tumor detected and extracted and image is given as output.

\section{Data Source}

In our work the images are collected from the website in internet and some samples are taken from the radiologists. The images collected from the radiologists are converted in to normal RGB images to DICOM converter. The Figure 2(a) and Figure 2(b) are the samples of MRI brain image without tumor and Figure 2(c) and Figure 2(d) are the samples of MRI brain image with tumor.

\section{Proposed Methodology}

The flow chart Figure 1 illustrates the procedure of tumor detection and segmentation. The images are acquired from radiologist and some images are downloaded from the brain tumor MRI database. Each image is pre-processing and applied various thresholding and morphological operations to segment and detects the tumor that is explained in more detail in the following sub sections.

\subsection{Image Pre-Processing and Enhancement}

Pre-processing stage removes the noise and also high frequency artifact seen in the image. It removes the patient name, age and other marks within in the image. You'll find 
so many methods available for pre-processing technique. After these stages the medical image is converted into standard image without noise, film artifacts and labels. This process is done by using median filter, high pass filter, label filter etc. In our experimentation we've used median filter for pre-processing and histogram equalization for image enhancement. Figure 3(a) will be original image without preprocessing. The Figure 3(b) and Figure 3(c) shows MRI image after pre-processing stage when label and high pass filters are applied.

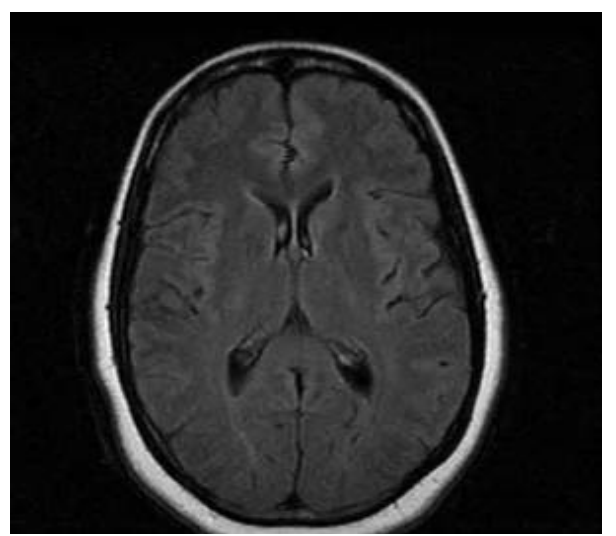

(a)

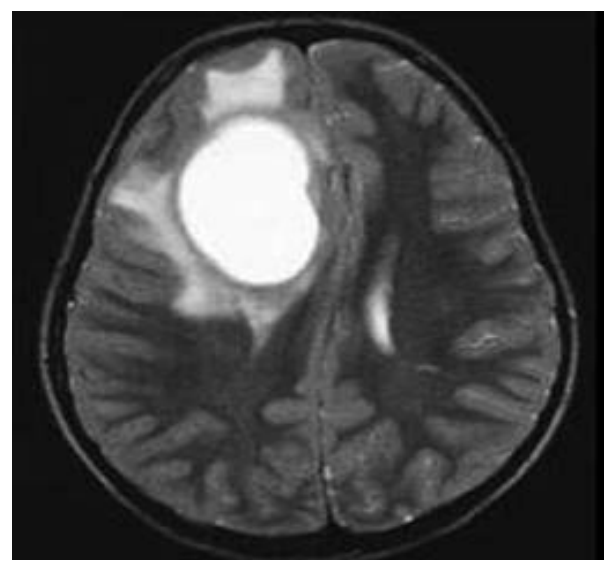

(c)

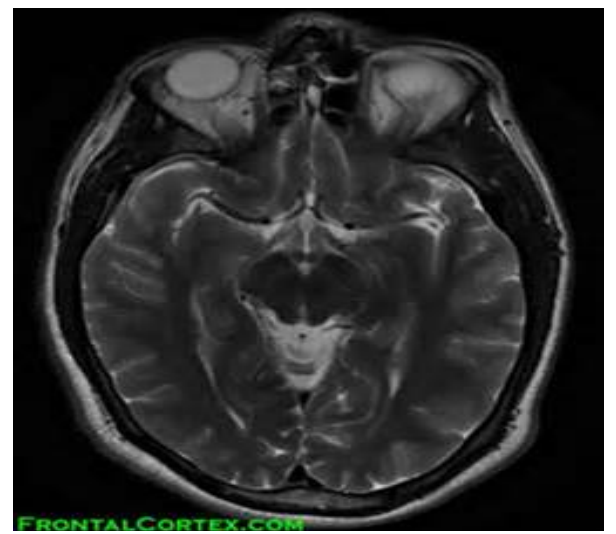

(b)

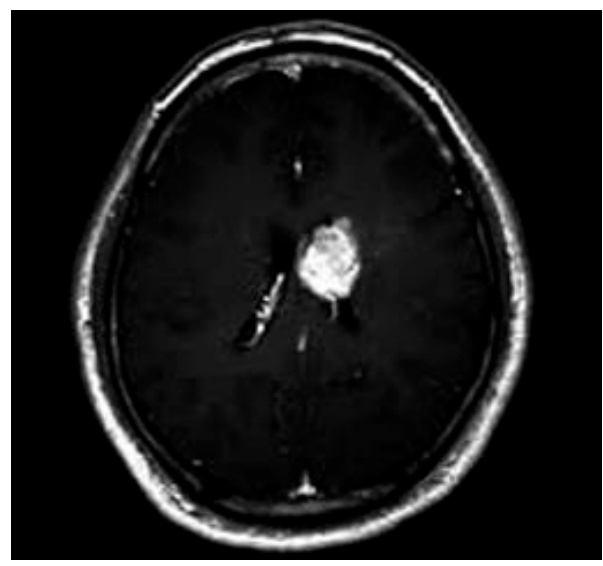

(d)

Figure 2. MRI images database with and without tumor.

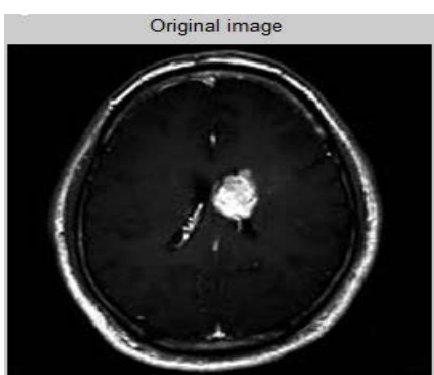

(a)

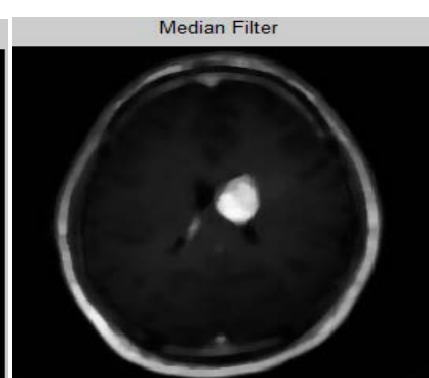

(b)

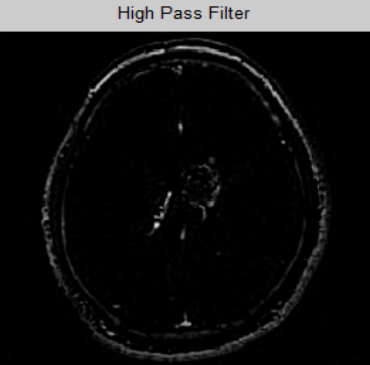

(c)

Figure 3. (a) Sample MRI brain image, (b) Pre-processed MRI brain image using median filter, (c) Pre-processed MRI brain image using high pass filter. 


\subsection{Histogram Equalization}

The histogram equalization is used to enhance the quality of the image. The continual probability density function and cumulative probability distribution functions are calculated. The Equation (1) is employed to calculate the cumulative probability distribution function. The frequency of occurrence of every gray value is calculated as well as the image is converted in a more useful form. The Figure 4(b) shows the output of histogram equalization of an image. From the histogram equalization the output image, we can easily notice that the contrast in the image is enhanced. The cumulative probability distribution function emerged by

$$
F_{u}(u)=\int_{0}^{u} P_{u}(u) \mathrm{d} u
$$

Here u may be the image pixel value where each pixel carries a continuous probability density function. $P_{u}(u)$ could be the continuous probability density function, $F_{u}(u)$ is the cumulative probability distribution function.

\subsection{Threshold Segmentation}

Segmentation is a division of the digital image into multiple parts and objective process is to simplify the representation of the image to what is more pronounced for the

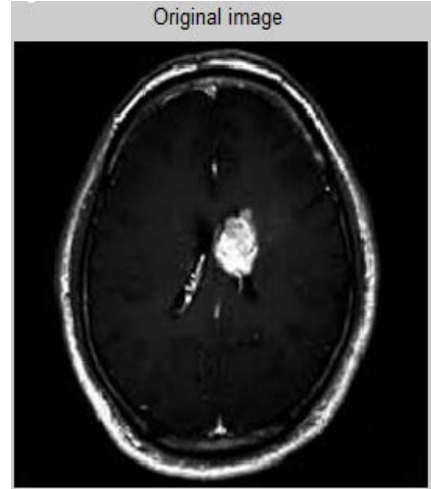

(a)

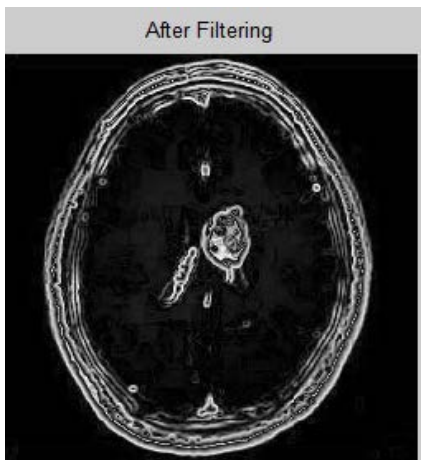

(e)

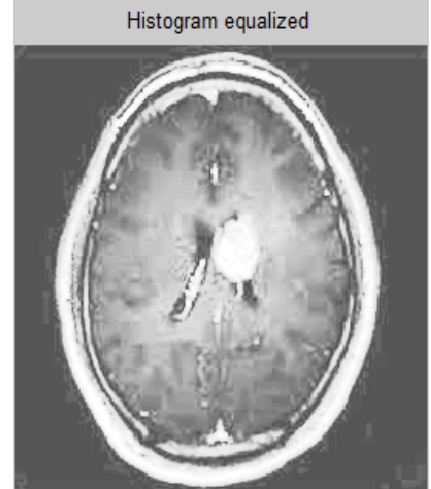

(b)

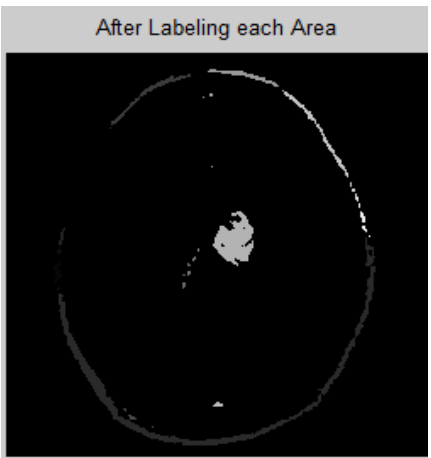

(f)

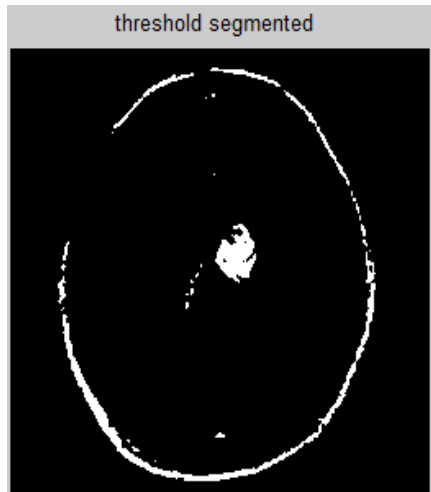

(c)

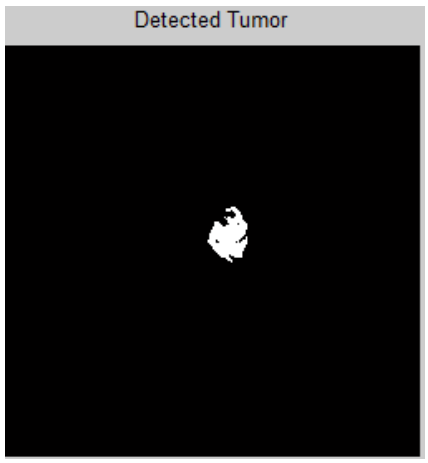

(g)

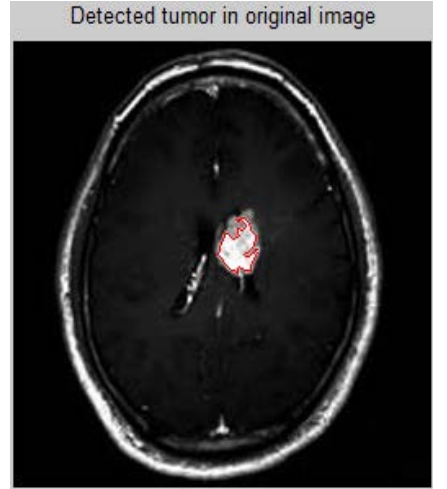

(d)

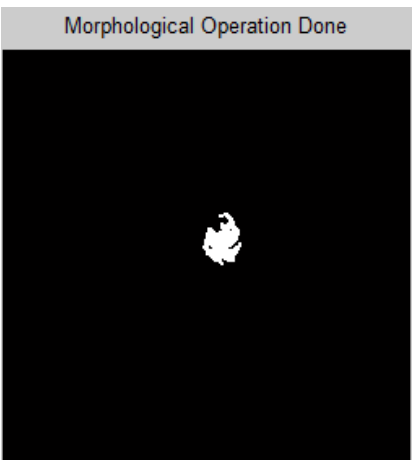

(h)

Figure 4. Tumor detection process. (a) Original MRI brain image, (b) Histogram equalization image, (c) threshold segmentation image, (d) Detected tumor in original image, (e) After filtering image, (f) After labeling each area image, (g) Detected tumor alone image, (h) Morphological operations image. 
analysis of the image. The threshold of an image is calculated using the Equation (2). The output of the threshold image is a binary image. Thresholding creates binary images from grey-level ones by turning all pixels below some threshold to zero and all pixels above that threshold to 1 . If $g(x, y)$ is a threshold version of $f(x, y)$ at some global threshold $\mathrm{T}$, then

$$
\begin{aligned}
& g(x, y)=1 \text { if } f(x, y)>T \\
& 0 \text { if } f(x, y)<T
\end{aligned}
$$

The output of thresholding segmentation is shown in Figure 4(c). $f(x, y)$ is the pixels in the input image and $g(x, y)$ is the pixels in the output image.

\subsection{Morphological Operations}

In medical image processing, we use mathematical morphological by means of identity and detect and extract significant image descriptors by using properties of the shape in an image. Morphological operations are the logical transformation established on comparison of pixel neighborhood with a specified pattern that is known as a structural element [8]. Here the morphological operations such as binary dilation, binary erosion are applied to the image. Binary closing and binary opening operations are applied to images using the Equations (3) and (4) to detected a tumor in original image which can be shown in Figure 4(d)

$$
\begin{aligned}
& A^{\circ} B=(A \Theta B) \oplus B \\
& A \cdot B=(A \oplus B) \Theta B
\end{aligned}
$$

The Figure 4(e) shows after filtering image, and the Figure 4(f) after labeling each area image.

\subsection{Tumor Detection}

Finally, this approach based on morphological operations detected the tumor area in MRI brain image which is shown in Figure 4(g) detected tumor image alone, and Figure 4(h) morphological operations image.

\section{Experimental Results}

The Experiment of detection of tumor in MRI brain image is carried out using thresholding segmentation and based on morphological operations and the snapshot of various stages of image processing is shown in the Figure 4 from a to h Each step indicates how detection of tumor is processed.

\section{Graphical User Interface Using the Novel Approach}

We used Graphical User Interface (Guide) especially for the effectiveness of the approach using two axes $x_{1}$ for input MR Brain image and $x_{2}$ for detected MR Brain image using novel approach as shown in Figure 5. 


\section{Overall Error and the Accuracy of the Approach}

Overall error is calculated from false alarm and miss alarm. Finally, accuracy of the approach system is measured with the help of overall error defined as

Overall Error $=$ False Alarm + Miss Alarm

Accuracy $=\frac{1-\text { Overall Error }}{\text { Image Dimension }} \times 100$

Finally we can show Overall Error in Figure 6 and The accuracy in Figure 7.

Kader_GUI

About Auther

\section{Brain Tumor Detection}
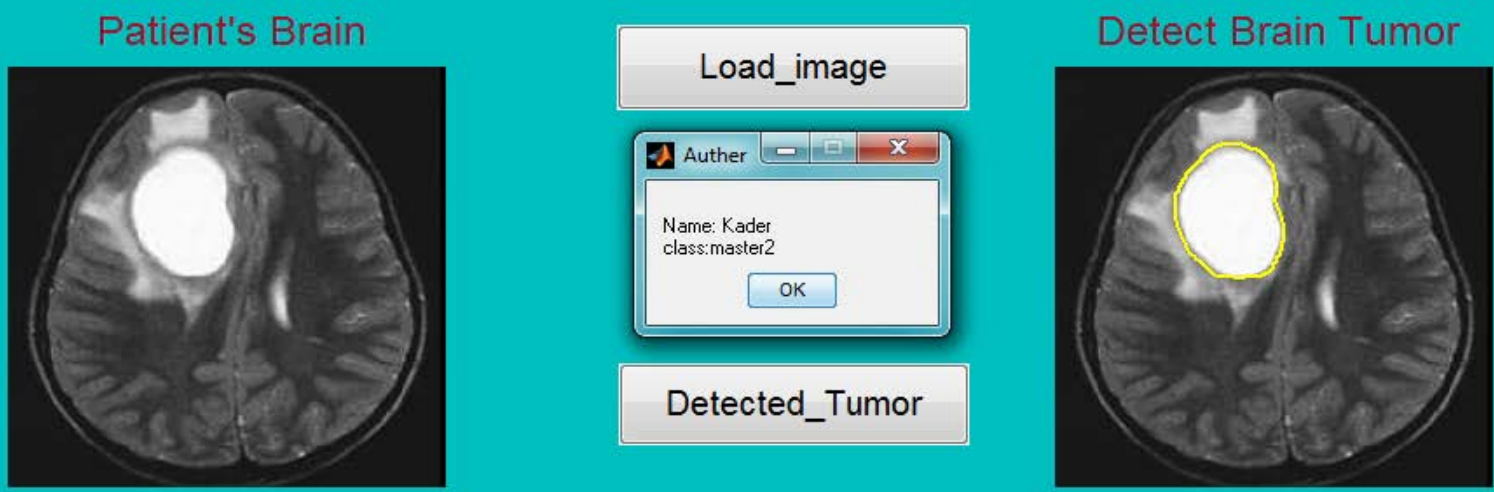

Figure 5. My guide for detection brain tumor using approaches.

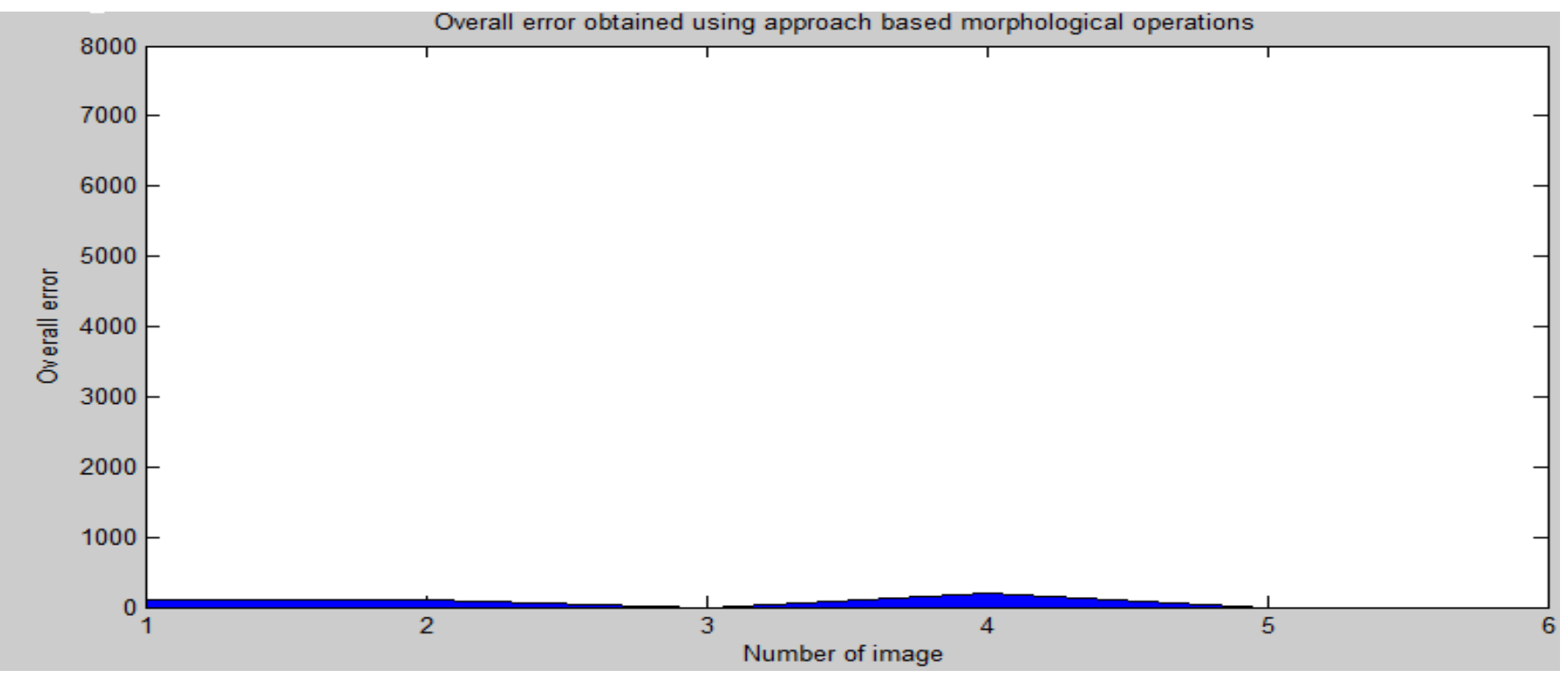

Figure 6. Overall error obtained using approach based morphological operations. 


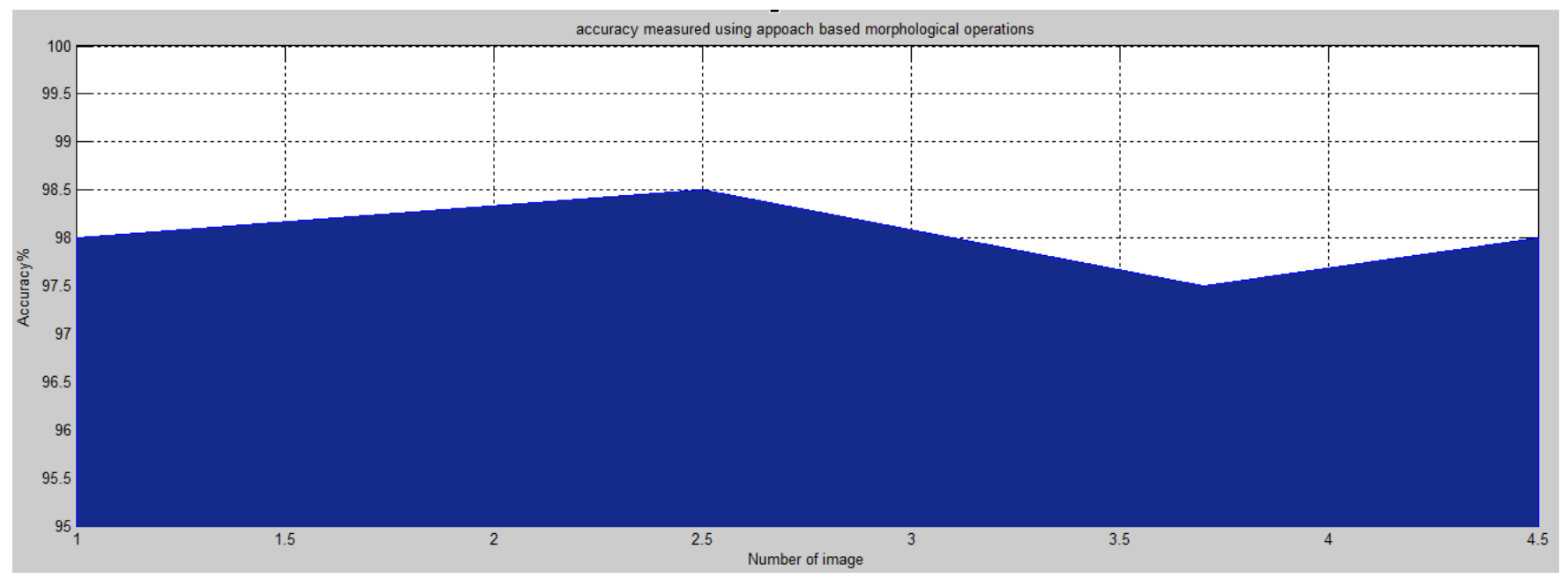

Figure 7. Accuracy obtained using approach based morphological operations.

\section{Conclusion}

In this paper, we presented a method for image acquisition, image pre-processing using median and high pass and label filtering, image enhancement using histogram equalization, segmentation using threshold and morphological operations therefore the detection of the tumor. Some of the features of the tumor are detected which will be helpful in medical applications. The future works involve the segmentation and detection of more images with more features which help in classifying several types of the tumors.

\section{Acknowledgements}

This work was supported in part by the natural science foundation of Hebei province under grant No: E2015202292 and No: E2015202050, high level talent support project in Hebei province under grant No: C2015005012, key research and development program under grant No: 15272002 and No: 15275704.

\section{References}

[1] Logeswari, T. and Karman, M. (2010) An Improved Implementation of Brain Tumor Detection Using Segmentation Based on Soft Computing. Journal of Cancer Research and Experimental Oncology, 2, 6-14.

[2] American Brain Tumor Association (2012) Facts and Statistics, 2012. http://www.abta.org/secure/about-brain-tumors-a-primer.pdf

[3] National Cancer Institute Dictionary of Cancer Terms. http://www.cancer.gov/publications/dictionaries/cancer-terms

[4] Devos, A. and Lukas, L. (2014) Does the Combination of Magnetic Resonance Imaging and Spectroscopic Imaging Improve the Classification of Brain Tumors? IEMBS $26^{\text {th }}$ Annual International Conference of the IEEE Engineering in Medicine and Biology Society, 1-5 September 2004, 407410.

[5] Natarajan, P., Krishnan, N., Kenkre, N.S., Nancy, S. and Singh, B.P. (2012) Tumor Detec- 
tion Using Threshold Operation in MRI Images. IEEE 2012.

[6] Gajanayak, G.M.N.R., Yapal, R.D. and Hewawithana, B. (2009) Comparison of Standard Image Segmentation Methods of Brain Tumors from 2D MR Images. ICIIS 2009, 28-31 December 2009. http://dx.doi.org/10.1109/iciinfs.2009.5429848

[7] Resmi, A.S. and Thomas, T. (2012) Automatic Segmentation Framework for Primary Tumors from Brain MRIs Using Morphological Filtering Techniques. $5^{\text {th }}$ BMEI, 2012.

[8] Thapaliya, K. and Kwon, G.R. (2012) Extraction of Brain Tumor Based on Morphological Operations. 8th (ICCM) 2012.

[9] Ahmed, F., Sharmin, P., Shahriar, B. and Sarwar, S. (2012) An Improved Image Denoising and Segmentation Approach for Detecting Tumor from 2-D MR Brain Images. IEEE 2012.

Submit or recommend next manuscript to SCIRP and we will provide best service for you:

Accepting pre-submission inquiries through Email, Facebook, LinkedIn, Twitter, etc.

A wide selection of journals (inclusive of 9 subjects, more than 200 journals)

Providing 24-hour high-quality service

User-friendly online submission system

Fair and swift peer-review system

Efficient typesetting and proofreading procedure

Display of the result of downloads and visits, as well as the number of cited articles

Maximum dissemination of your research work

Submit your manuscript at: http://papersubmission.scirp.org/

Or contact jbise@scirp.org 